\title{
Gravitinos from gravitational collapse
}

\author{
J. A. Grifols, E. Massó, and R. Toldrà \\ Grup de Física Teòrica and Institut de Física d'Altes Energies, Universitat Autònoma de Barcelona, \\ 08193 Bellaterra, Barcelona, Spain \\ (Received 1 August 1997; published 21 November 1997)
}

\begin{abstract}
We reanalyze the limits on the gravitino mass $m_{3 / 2}$ in superlight gravitino scenarios derived from arguments on energy loss during gravitational collapse. We conclude that the mass range $10^{-6} \mathrm{eV} \leqslant m_{3 / 2} \leqslant 2.3 \times 10^{-5} \mathrm{eV}$ is excluded by SN 1987A data. In terms of the scale of supersymmetry breaking $\Lambda$, the range $70 \mathrm{GeV} \leqslant \Lambda$ $\leqslant 300 \mathrm{GeV}$ is not allowed. [S0556-2821(98)01601-4]
\end{abstract}

PACS number(s): 14.80.Ly, 04.65.+e, 97.60.Bw

In a wide class of supergravity models with supersymmetry (SUSY) breaking $\Lambda$ in the $\mathrm{TeV}$ range, the gravitino can be very light:

$$
m_{3 / 2}=2.5 \times 10^{-4} \mathrm{eV}(\Lambda / 1 \mathrm{TeV})^{2} .
$$

Indeed, models where gauge interactions mediate the breakdown of supersymmetry [1], models where an anomalous U(1) gauge symmetry induces SUSY breaking [2], and noscale models are all examples of models where a superlight gravitino can be accommodated [3]. In all of them, the gravitino is the lightest supersymmetric particle (LSP) and, furthermore, its couplings to matter and radiation are inversely proportional to its mass. Therefore, one may expect interesting phenomenology [4]. Bounds on the gravitino mass, or equivalently on the scale $\Lambda$ have been given in the context of those models by various authors and have been extracted from different physical systems. In fact, the limits come from as distinct areas as the anomalous magnetic moment of the muon [5], primordial nucleosynthesis [6] or stellar energy drain arguments [7]. In recent papers [8], it has been noted that the amplitudes for gravitino processes that were used in deriving some of the constraints had an incorrect energy behavior. In particular, the supernova (SN) bounds deduced in Ref. [9] using the effective couplings given explicitly by Gherghetta [10] are invalid as pointed out by Luty and Ponton [11]. These authors, however, when reexamining the limits coming from the SN 1987A explosion, use an incorrect abundance of positrons in the core, do not discuss gravitino bremsstrahlung, and misidentify the main source of opacity in the stellar core. The purpose of the present paper is thus to redo the analysis that renders the bounds on $m_{3 / 2}$ (or $\Lambda$ ) following from SN collapse. Since SN considerations gave the best limits on $\Lambda$ up to now [11], this is not an empty exercise.

The relevant piece in the effective Lagrangian is the derivative coupling of the Goldstino $\chi$ to photons:

$$
\delta L_{e f f}=(e / 2)\left(M / \Lambda^{2}\right)^{2} \partial^{\mu} \chi \sigma^{\nu} \bar{\chi} F_{\mu \nu}+\text { H.c. }
$$

with $F_{\mu \nu}$, the electromagnetic field strength and $M$ is a mass that depends on the supersymmetry breaking model. In gauge-mediated models, $M \sim m_{\tilde{L}} / 4 \pi$, where $m_{\tilde{L}}$ is the lefthanded slepton mass. Given that gravitino pairs are mainly produced via one-photon interactions, the sources of grav- itino luminosity in stars are, in principle, gravitino bremsstrahlung in neutron-proton scattering, pair production in electron-positron annihilation and plasmon decay into gravitinos. The energy-loss rate (per unit volume) via $p n \rightarrow p n \widetilde{G} \widetilde{G}$ is

$$
\begin{aligned}
Q= & \int \frac{d^{3} k_{1}}{(2 \pi)^{3} 2 k_{1}^{0}} \frac{d^{3} k_{2}}{(2 \pi)^{3} 2 k_{2}^{0}} \prod_{i=1}^{4} \frac{d^{3} p_{i}}{(2 \pi)^{3} 2 p_{i}^{0}} f_{1} f_{2}\left(1-f_{3}\right) \\
& \times\left(1-f_{4}\right)(2 \pi)^{4} \delta^{4}\left(P_{f}-P_{i}\right) \sum_{\text {spins }}\left|M_{f i}\right|^{2}\left(k_{1}^{0}+k_{2}^{0}\right),
\end{aligned}
$$

where $\left(p^{0}, \vec{p}\right)_{i}$ are the four-momenta of the initial and final state nucleons, $\left(k^{0}, \vec{k}\right)_{1,2}$ are the four-momenta of the gravitinos and $f_{1,2}$ are the Fermi-Dirac distribution functions for the initial proton and neutron and $\left(1-f_{3,4}\right)$ are the final Pauli blocking factors for the final proton and neutron. The squared matrix element can be factorized as follows:

$$
\sum_{\text {spins }}\left|M_{f i}\right|^{2}=(2 \pi)^{2} \alpha^{2}\left(M / \Lambda^{2}\right)^{4} N_{\mu \nu} G_{3 / 2}^{\mu \nu}
$$

where $N_{\mu \nu}$ is the nuclear [one pion exchange (OPE)] tensor and $G_{3 / 2}^{\mu \nu}$ is the gravitino tensor in the matrix element squared. The factor $N_{\mu \nu}$ is common to any bremsstrahlung process involving nucleons. It appears, e.g., in neutrino bremsstrahlung calculations and in axion bremsstrahlung calculations, and is given explicitly in Ref. [12]. On the other hand, $G_{3 / 2}^{\mu \nu}$ is a tensor specific to gravitino bremsstrahlung. It reads

$$
G_{3 / 2}^{\mu \nu}=k_{1}^{\mu} k_{2}^{\nu}+k_{2}^{\mu} k_{1}^{\nu}-k_{1} \cdot k_{2} g^{\mu \nu} .
$$

The integration of $N_{\mu \nu}$ over the phase space of the nucleons can be performed explicitly and the details can be found again in Raffelt's book [12]. When we contract the result with the gravitino tensor $G_{3 / 2}$ and perform the integrations over gravitino momenta to complete the energy depletion rate, we are led to the following emissivity:

$$
Q_{b r e m s}^{N D}=\left(8192 / 385 \pi^{3 / 2}\right) \alpha^{2} \alpha_{\pi}^{2}\left(M / \Lambda^{2}\right)^{4} Y_{e} n_{B}^{2} T^{11 / 2} / m_{p}^{5 / 2}
$$

for nondegenerate and nonrelativistic nucleons $\left(\alpha_{\pi}\right.$ is the pionic fine-structure constant, $n_{B}$ is the number density of 
baryons, and $Y_{e}$ is the mass fraction of protons). However, nucleons are moderately degenerate in the $\mathrm{SN}$ core. The emissivity in the (extreme) degenerate case is calculated to be

$$
Q_{b r e m s}^{D}=\left(164 \pi^{3} / 4725\right) \alpha^{2} \alpha_{\pi}^{2}\left(M / \Lambda^{2}\right)^{4} p_{F} T^{8}
$$

with $p_{F}$, the Fermi momentum of the nucleons. Numerically, for the actual conditions of the star, both emissivities differ by less than an order of magnitude (about a factor of $3)$. Since the actual emissivity interpolates between these two values, we shall adopt the smallest of the two (i.e., $Q_{b r e m s}^{N D}$ ) to make our (conservative) estimates. We turn next to the annihilation process.

The energy loss for the process $e^{+}\left(p_{1}\right)+e^{-}\left(p_{2}\right)$ $\rightarrow \widetilde{G}\left(k_{1}\right)+\widetilde{G}\left(k_{2}\right)$ can be calculated along similar lines as above. The spin averaged matrix element squared is, in this case,

$$
\sum_{\text {spins }}\left|M_{f i}\right|^{2}=(2 \pi)^{2} \alpha^{2}\left(M / \Lambda^{2}\right)^{4} E_{\mu \nu}\left(p_{1}, p_{2}\right) G_{3 / 2}^{\mu \nu}\left(k_{1}, k_{2}\right),
$$

where $E_{\mu \nu}\left(p_{1}, p_{2}\right)$ equals formally the tensor $G_{3 / 2}^{\mu \nu}$ in Eq. (5) with $k_{1}, k_{2}$ replaced by $p_{1}, p_{2}$. The luminosity then is found to be

$$
Q_{a n n}=8 \alpha^{2}\left(M / \Lambda^{2}\right)^{4} T^{4} e^{-\mu / T} \mu^{5} b(\mu / T) / 15 \pi^{3}
$$

with $\quad b(y) \equiv(5 / 6) e^{y} y^{-5}\left(F_{5}^{+} F_{4}^{-}+F_{4}^{+} F_{5}^{-}\right) \quad$ where $\quad F_{m}^{ \pm}(y)$ $=\int_{0}^{\infty} d x x^{m-1} /\left(1+e^{x \pm y}\right)(\mu$ is the chemical potential of the electrons). The function $b(y) \rightarrow 1$ in the degenerate limit. Finally, our estimate of the plasmon decay luminosity is

$$
Q_{P}=16 \zeta(3) \alpha^{4} T^{3} \mu^{6}\left(M / \Lambda^{2}\right)^{4} / 81 \pi^{5}
$$

(where only transverse plasmons have been taken into account).

Taken at face value, the bremsstrahlung rate is the largest of the three. However, $Q_{\text {brems }}$ is overestimated since we did not consider multiple scattering effects which are present in a dense medium [12]. Indeed, as for the axion case [12], the gravitino bremsstrahlung rate probably saturates around $20 \%$ nuclear density and this should be taken into account when evaluating Eq. (6). If we use now the values $T=50 \mathrm{MeV}$, $\mu=300 \mathrm{MeV}$, and $Y_{e}=0.3$, Eqs. (6) (with $n_{B} \sim 0.2 n_{n u c}$ ), (9), and (10) give

$$
Q_{\text {ann }}: Q_{\text {brems }}: Q_{P} \approx 1.2 \times 10^{3}: 3 \times 10^{2}: 1 .
$$

Therefore, a limit on $\Lambda$ will follow from the requirement that $L_{3 / 2} \approx V Q_{a n n}$ ( $V$ is the volume of the stellar core) should not exceed $10^{52} \mathrm{ergs} / \mathrm{s}$. This constraint on the gravitino luminosity $L_{3 / 2}$ implies, in turn,

$$
\begin{aligned}
\Lambda \geqslant & 300 \mathrm{GeV}(M / 43 \mathrm{GeV})^{1 / 2}(T / 50 \mathrm{MeV})^{11 / 16} \\
& \times\left(R_{c} / 10 \mathrm{~km}\right)^{3 / 8}
\end{aligned}
$$

or, using Eq. (1),

$$
m_{3 / 2} \geqslant 2.3 \times 10^{-5} \mathrm{eV} .
$$

Of course, the previous calculation makes sense only if gravitinos, once produced, stream freely out of the star without rescattering. That they actually do so, for $\Lambda \geqslant 300 \mathrm{GeV}$, can be easily checked by considering their mean-free path in the core. The main source of opacity for gravitinos is the elastic scattering off the Coulomb field of the protons:

$$
\lambda=1 / \sigma n=\left(4 / \pi \alpha^{2}\right) Y_{e}^{-1} \rho^{-1} m_{p}^{-1}\left(\Lambda^{2} / M\right)^{4} .
$$

The thermally averaged cross section for elastic gravitino scattering on electrons is roughly a factor $T \mu / m_{p}^{2}$ smaller than that on protons and thus it does not contribute appreciably to the opacity. Putting numbers in Eq. (14) we find

$$
\lambda \simeq 1.4 \times 10^{7} \mathrm{~cm}(43 \mathrm{GeV} / M)^{4}(\Lambda / 300 \mathrm{GeV})^{8} .
$$

On the other hand, the calculation of $Q$ breaks down for $\lambda \leqslant 10 \mathrm{~km}$, i.e., for $\Lambda \leqslant 220 \mathrm{GeV}$, when gravitinos are trapped in the SN core. In this case, gravitinos diffuse out of the dense stellar interior and are thermally radiated from a gravitino sphere $R_{3 / 2}$. Because in this instance the luminosity is proportional to $T^{4}$, only for a sufficiently large $R_{3 / 2}$ (where the temperature is correspondingly lower), the emitted power will fall again below the nominal $10^{52} \mathrm{erg} / \mathrm{s}$. Consequently, gravitino emission will be energetically possible, if $\Lambda$ is small enough. The gravitino-sphere radius can be computed from the requirement that the optical depth

$$
\tau=\int_{R}^{\infty} d r / \lambda(r)
$$

be equal to $2 / 3$ at $R=R_{3 / 2}$. Here, $\lambda(r)$ is given in Eq. (14) with the density profile ansatz:

$$
\rho(r)=\rho_{c}\left(R_{c} / r\right)^{m}
$$

with $\rho_{c}=8 \times 10^{14} \mathrm{~g} / \mathrm{cm}^{3}, R_{c}=10 \mathrm{~km}$ and $m=5-7$ and which satisfactorily parametrizes the basic properties of $\mathrm{SN}$ 1987A [13]. An explicit calculation renders

$$
R_{3 / 2}=R_{c}\left[\left(8 Y_{e} / 3 \pi \alpha^{2}\right)\left(\Lambda^{2} / M\right)^{4}(m-1) / \rho_{c} R_{c} m_{p}\right]^{1 / 1-m} .
$$

Stefan-Boltzmann's law implies for the ratio of gravitino to neutrino luminosities,

$$
L_{3 / 2} / L_{\nu}=\left(R_{3 / 2} / R_{\nu}\right)^{2}\left[T\left(R_{3 / 2}\right) / T\left(R_{\nu}\right)\right]^{4},
$$

where $R_{\nu}$ is the radius of the neutrinosphere. To proceed further we use the temperature profile:

$$
T=T_{c}\left(R_{c} / r\right)^{m / 3}
$$

which is a consequence of Eq. (17) and the assumption of local thermal equilibrium. Now, taking $m=7$ [14], we obtain

$$
L_{3 / 2} / L_{\nu}=\left(R_{\nu} / R_{c}\right)^{22 / 3}\left[\left(16 Y_{e} / \pi \alpha^{2}\right)\left(\Lambda^{2} / M\right)^{4} / \rho_{c} R_{c} m_{p}\right]^{11 / 9} .
$$

By demanding that $L_{3 / 2} \leqslant 0.1 L_{\nu}$ and using $R_{\nu} \simeq 30 \mathrm{~km}$, we obtain

$$
\Lambda \leqslant 70 \mathrm{GeV} .
$$


This in turn implies $m_{3 / 2} \leqslant 10^{-6} \mathrm{eV}$. Since, on the other hand, the anomalous magnetic moment of the muon already requires $m_{3 / 2}$ to be larger than $\sim 10^{-6} \mathrm{eV}[5,15]$, we are forced to conclude that

$$
\Lambda \geqslant 300 \mathrm{GeV}
$$

or, equivalently,

$$
m_{3 / 2} \geqslant 2.3 \times 10^{-5} \mathrm{eV} \text {. }
$$

In conclusion, we have carefully rederived the bounds on the superlight gravitino mass (i.e., the SUSY scale $\Lambda$ ) that follow from SN physics. These limits are completely general in the sense that they do not rely on other particles in a given particular model being light. Should other particles such as the scalar partners of the Goldstino also be light, then the resulting bounds are necessarily tighter. In such clearly less general frame, constraints have also been derived in the literature [16] that are not subject to the criticisms mentioned in the beginning of this paper. They are much stronger then the ones given here and typically give $\Lambda \geqslant 300 \mathrm{TeV}$ (or, $m_{3 / 2} \geqslant 50 \mathrm{eV}$ ) from stellar (e.g., the Sun) evolution arguments, provided $m_{3 / 2} \leqslant 1 \mathrm{keV}$ (e.g., $T_{\odot}$ ).

This work was partially supported by the CICYT Research Projects AEN95-0815 and AEN95-0882 and the Theoretical Astroparticle Network under the EEC Contract No. CHRX-CT93-0120 (Direction Generale 12 COMA).
[1] M. Dine, A. E. Nelson, Y. Nir, and Y. Shirman, Phys. Rev. D 53, 2658 (1996); M. Dine, A. E. Nelson, and Y. Shirman, ibid. 51, 1362 (1995).

[2] P. Binetruy and E. Dudas, Phys. Lett. B 389, 303 (1996); G. Dvali and A. Pomarol, Phys. Rev. Lett. 77, 3728 (1996); R. N. Mohapatra and A. Riotto, Phys. Rev. D 55, 4262 (1997).

[3] J. Ellis, K. Enqvist, and D. V. Nanopoulos, Phys. Lett. 147B, 99 (1984).

[4] J. Kim et al., Report No. ACT-11/97; CERN-TH/97-120; CTP-TAMU-30/97; DOE/ER/40717-44, hep-ph/9707331, 1997.

[5] A. Mendez and F. X. Orteu, Nucl. Phys. B256, 181 (1985).

[6] P. Fayet, Proceedings of the 16th Rencontre de Moriond, edited by T. Tran Thanh Van (Editions Frontieres, Les Arcs, 1981).

[7] M. Nowakowski, S. D. Rindani, Phys. Lett. B 348, 115 (1995).

[8] A. Brignole, F. Feruglio, and F. Zwirner, Report No. CERNTH/96-369; DFPD-96/TH/68, hep-ph/9703286, 1997; M. A.
Luty and E. Ponton, Report No. UMD-PP-97-116, hep-ph/9706268, 1997.

[9] J. A. Grifols, R. N. Mohapatra, and A. Riotto, Phys. Lett. B 401, 283 (1997).

[10] T. Gherghetta, Nucl. Phys. B485, 25 (1997).

[11] M. A. Luty and E. Ponton, Report No. UMD-PP-97-116, hep-ph/9706268, 1997.

[12] G. G. Raffelt, Stars as Laboratories for Fundamental Physics (Chicago University Press, Chicago, 1996).

[13] M. S. Turner, Phys. Rev. Lett. 60, 1797 (1988).

[14] R. N. Mohapatra and I. Z. Rothstein, Phys. Lett. B 247, 593 (1990); J. A. Grifols and E. Masso, Nucl. Phys. B331, 244 (1990).

[15] F. Ferrer and J.A. Grifols, Phys. Rev. D 56, 7466 (1997); T. Li, J. L. Lopez, and D. V. Nanopoulos, Report No. CTPTAMU-23/97; DOE/ER/40717-43, hep-ph/9704439, 1997.

[16] J. A. Grifols, R. N. Mohapatra, and A. Riotto, Phys. Lett. B 400, 124 (1997). 